\title{
Early growth response factor-1 DNA enzyme 1 inhibits the formation of abdominal aortic aneurysm in rats
}

\author{
SHI WANG ${ }^{1}$, HAIPENG DONG ${ }^{2}$, CHENGWEI LIU ${ }^{3}$, GUICHAO XU ${ }^{3}$, \\ XINHUA HU ${ }^{4}$, YICHUAN FAN ${ }^{4}$ and LITING CHEN ${ }^{5}$

\begin{abstract}
${ }^{1}$ Department of Stomatology, The First Affiliated Hospital of Jiamusi University, Jiamusi, Heilongjiang 154002;
${ }^{3}$ Division of Vascular Surgery, The First Affiliated Hospital of Jiamusi University, Jiamusi, Heilongjiang 154002;

${ }^{4}$ Department of Surgery, First Affiliated Hospital, China Medical University, Shenyang, Liaoning 110000;

${ }^{5}$ Department of Emergency, Affiliated Hospital of Beihua University, Jilin City, Jilin 132000, P.R. China
\end{abstract} \\ ${ }^{2}$ Department of Cardiothoracic Vascular Surgery, Affiliated Hospital of Beihua University, Jilin City, Jilin 132000;
}

Received August 30, 2017; Accepted April 24, 2018

DOI: $10.3892 /$ etm.2018.6139

\begin{abstract}
The aim of the present study was to characterize the effects of early growth response factor-1 DNA enzyme (EDRz) in a rat abdominal aortic aneurysm (AAA) model to determine the mechanism by which EDRz inhibits AAA and affects the formation of AAA by regulating the activity of matrix metalloproteinase (MMP)-2 and MMP-9. EDRz was transfected into the abdominal aorta of rats using the jetPRIME transfection reagent following infusion with elastase. Fluorescent microscopy, hematoxylin and eosin staining, ultrastructural analysis, reverse transcription-quantitative polymerase chain reaction, western blotting and immunohistochemical analysis were performed to characterize the response to EDRz. The EDRz group showed minimal aneurysm formation when compared with the control group, with significantly lower aortic diameter expansion $(2.5 \pm 0.1$ vs. $3.5 \pm 0.1 \mathrm{~mm}$; $\mathrm{P}<0.05)$. Early growth response factor 1 (Egr-1) mRNA and protein levels were significantly decreased in the EDRz group, as expected. The decrease in Egr-1 was accompanied by decreases in the mRNA and protein levels of MMP-2 and MMP-9 $(\mathrm{P}<0.05)$. Transfection of the Egr-1 specific synthetic DNA enzyme EDRz significantly reduced AAA following elastase infusion in rats, at least in part due to the decreased expression of downstream MMP-2 and MMP-9.
\end{abstract}

\section{Introduction}

Abdominal aortic aneurysm (AAA) is a serious disease with high morbidity and high mortality, and its incidence has

Correspondence to: Dr Chengwei Liu, Division of Vascular Surgery, The First Affiliated Hospital of Jiamusi University, 348 Dexiang Street, Jiamusi, Heilongjiang 154002, P.R. China E-mail: docchengweiliu@163.com

Key words: abdominal aortic aneurysm, matrix metalloproteinases, early growth response factor 1 DNA enzyme, elastase increased with lifestyle changes and aging. Many factors affect the occurrence and development of AAA, such as hypertension, peripheral arterial disease, and smoking, among others. Thus, early detection and early treatment as well as joint screening play an important role in the occurrence and development of AAA $(1,2)$. However, no drugs or methods have been developed to inhibit the formation and development of AAAs in an early stage. AAA is pathologically characterized by chronic inflammation of the aortic wall, degradation of the extracellular matrix (3), extensive destruction and re-formation of the medial layer during neovascularization, and attenuation of vascular smooth muscle cells (4). These changes destroy medial membranes and promote aortic aneurysm expansion. Thus, the aorta gradually expands and eventually breaks down.

Early growth response factor 1 (Egr-1) is a zinc finger transcription factor and member of the early gene family. Egr-1 participates in various pathophysiologic processes, such as cell proliferation, differentiation, and apoptosis (5), inflammatory reaction (6), thrombosis (7), and extracellular matrix degradation and synthesis (8). Egr-1 expression can be rapidly induced by various stimulating factors, such as growth factors, proinflammatory cytokines, lipopolysaccharides, hypoxia, shear stress, and vascular damage. Once activated, Egr-1 controls the expression of several genes implicated in the pathogenesis of AAA, indicating that this transcription factor represents a key molecular target for controlling the formation of AAA. Bioinformatics analysis showed that Egr-1 is a key transcription factor in the formation and pathogenesis of AAA (9). Egr-1 has been also observed to be involved in thrombus formation and the inflammatory pathogenesis of AAA (10). The role of Egr-1 as a mechanical stress-response gene causing aneurysm formation was also demonstrated (11). Thus, Egr-1 is considered as a target for AAA prevention and control.

Early growth response factor-1 DNA enzyme (EDRz) is a small single-stranded DNA fragment with enzymatic activity that can specifically cut Egr-1, inhibit the protein expression of Egr-1, and block the expression of other Egr-1-regulated genes. As a biological tool, EDRz can specifically block gene expression. However, it is unclear whether EDRz inhibits AAA or the expression of other genes related to Egr-1. 
In the present study, EDRz was introduced into rats with AAA, and the effect of EDRz on AAA formation was examined. In addition, the expression of Egr-1 and matrix metalloproteinase (MMP)-2, MMP-9 were examined after introducing EDRz into vessels. This study aimed to examine the inhibitory effects of EDRz on the formation of AAA and verify the feasibility of this enzyme for AAA gene therapy.

\section{Materials and methods}

Construction of EDRz. The DNA enzyme EDRz was synthesized by Sangon Biotech Co. (Shanghai, China) according to the sequence published in GenBank. Fig. 1 shows the EDRz sequence as follows: 5'-CCGCTGCCAGGCTAGCTACAA CGACCCGGACGT-3'. The 3' termini of the oligonucleotides were protected from exonucleases by phosphorothioate linkage. Fluorescence microscopy was applied to determine the nucleotide distribution by using marked 5'-end carboxyl fluorescein. A total of $495 \mu \mathrm{g}$ of EDRz was added to $80 \mu \mathrm{l}$ of diethyl pyrocarbonate solution $(1: 1,000)$ and mixed after centrifugation. Next, $120 \mu 1$ of jetPRIME (Polyplus-transfection SA, Illkirch, France), $32 \mu \mathrm{l}$ of $1 \mathrm{mM} \mathrm{MgCl}_{2}$, and $568 \mu \mathrm{l}$ of $30 \%$ F-127 pluronic gel (Sigma-Aldrich; Merck KGaA, Darmstadt, Germany) were added. This $800 \mu$ l solution was shock-mixed and stored at $4^{\circ} \mathrm{C}(7)$.

Ninety male SD rats (Liaoning Changsheng Biotechnology, Dalian, China) weighing 250-300 g were used in this study. This study was carried out in strict accordance with the recommendations in the Guide for the Care and Use of Laboratory Animals of the National Institutes of Health. The animal use protocol was been reviewed and approved by the Institutional Animal Care and Use Committee (IACUC) of Jiamusi University. The rats were randomly divided into three groups: control group (only elastase perfusion), jetPRIME group (elastase perfusion + jetPRIME transfection reagent), and EDRz group (elastase perfusion + jetPRIME transfection reagent + Egr-1 DNA enzyme). Thirty rats were included in each group. The rats were anesthetized with $3 \%$ pentobarbital sodium (40 mg/kg, intraperitoneal injection). The skin was prepared and disinfected, and then the rodents were fixed on an operating table in the supine position. A SXP-1C-type microscope (x10 magnification; Smoif, Shanghai, China) was used for sterile surgery. The inferior vena cava and abdominal aorta were separated (Fig. 2). The abdominal aortic trunk with a length of about approximately $1 \mathrm{~cm}$ located inferior to the left renal vein was separated and the infrarenal aortic diameter was measured. To block the blood flow on the upper part of this segment, we inserted a PE10 catheter (Smiths Medical, Minneapolis, MN, USA) into the left common iliac artery to the separated abdominal aorta. The lower part of the aorta was ligated temporarily to create a closed lumen, and $1 \mathrm{ml}(20 \mu)$ of elastase (Beijing Solarbio Science and Technology Co., Ltd., Beijing, China) was perfused for $20 \mathrm{~min}$. For the EDRz group, $20 \mu 1$ of the mixture containing DEPC, jetPRIME, $\mathrm{MgCl}_{2}$, EDRz, and F-127 pluronic gel was used and evenly coated on the peri-adventitial of the elastase perfused abdominal aorta. The specimens were collected after 28 days.

Detecting transfection of EDRz. The specimens were fixed and dehydrated in a sucrose gradient. The sections were embedded and sliced into $5-\mu \mathrm{m}$-thick sections with a constant freezing microtome. Transfection of EDRz was observed under a fluorescence microscope.

Ultrastructural analysis. Animal pressure perfusion was fixed with $3 \%$ glutaraldehyde solution in $0.1 \mathrm{M}$ of phosphate buffer ( $\mathrm{pH} 7.4$ ) via a catheter for $30 \mathrm{~min}$ at $20^{\circ} \mathrm{C}$ with a pressure of $100 \mathrm{mmHg}$. Specimens were dehydrated with alcohol and embedded in EPON. The sections were stained with lead citrate and uranyl acetate and observed with a transmission electron microscope.

Hematoxylin and eosin $(H \& E)$ staining. AAA specimens were fixed in $4 \%$ polyformaldehyde for at least $24 \mathrm{~h}$, and the conventional paraffin-embedded sections were cut into $5-\mu \mathrm{m}$-thick slices. Changes in the morphological characteristics of the aortic wall were observed by $\mathrm{H} \& \mathrm{E}$ staining.

Reverse transcription-quantitative polymerase chain reaction $(R T-q P C R)$. Total RNA was extracted with TRIzol reagent (Invitrogen; Thermo Fisher Scientific, Inc., Waltham, MA, USA) from the medial membrane of AAA, and the purity and concentration of RNA were determined using a NanoDrop1000 (NanoDrop; Thermo Fisher Scientific, Inc.; Wilmington, DE, USA). The RNA was reverse-transcribed into cDNA using the PrimeScript RT reagent Kit (Takara Bio, Inc., Otsu, Japan). qPCR was performed using SYBR Premix Ex Taq $^{\mathrm{TM}}$ II (Takara Bio, Inc.) on an ABI 7500 Real-Time PCR System (Applied Biosystems; Thermo Fisher Scientific, Inc., Waltham, MA, USA). The qPCR parameters were as follows: $95^{\circ} \mathrm{C}$ of denaturation for $30 \mathrm{sec}, 95^{\circ} \mathrm{C}$ for $5 \mathrm{sec}$, and $60^{\circ} \mathrm{C}$ for $30 \mathrm{sec}$ for a total of 40 times. The sequences of the primers were as follows: Egr-1: 5'-CAGGAGTGATGA ACGCAAGA-3' (forward) and 5'-GGGGATGGGTAGGAA GAGAG-3' (reverse). MMP-2: 5'-GATACAGGTGTGCCA AGGTG-3' (forward) and 5'-AAAGGGCAAACAAAG CAAAC-3' (reverse). MMP-9: 5'-CTGCAGTGCCCTTGA ACTAA-3' (forward) and 5'-TATCCGGCAAACTAGCTC CT-3' (reverse); $\beta$ actin: 5'-TGTCACCAACTGGGACGA TA-3' (forward) and 5'-GGGGTGTTGAAGGTCTCAAA-3' (reverse). The expression of the target gene was determined using $\beta$-actin as a reference using the $2^{-\Delta \Delta C q}$ method. Samples were examined in triplicate.

Western blotting. Total protein was extracted from the frozen medial membrane of the AAA with Lysis Buffer RIPA (Beyotime Institute of Biotechnology, Beijing, China). The protein concentration was determined using the BCA (Science Fdbiao, China) protein concentration assay kit. Protein extracts were separated by sodium dodecyl sulfate polyacrylamide gel electrophoresis in 12 sodium dodecyl sulfate and transferred to a polyvinylidene fluoride membrane. Skim milk or bovine serum albumin was added to Tris-buffered saline, blocked for $1 \mathrm{~h}$, and then incubated with Egr-1 antibody [EGR1 (15F7) Rabbit mAb, cat. no. 4153, 1:1,000; Cell Signaling Technology, Inc., Danvers, MA, USA], MMP-2 (MMP-2 Polyclonal Antibody, cat. no. A6247, 1:1,000; ABclonal Technology, Woburn, MA, USA), MMP-9 (MMP-9 Polyclonal Antibody, cat. no. A11147, 1:1,000; ABclonal Technology), and $\beta$-actin (cat. no. TA-09, 1:2,000; ZSGB-BIO, Beijing, China) antibody 


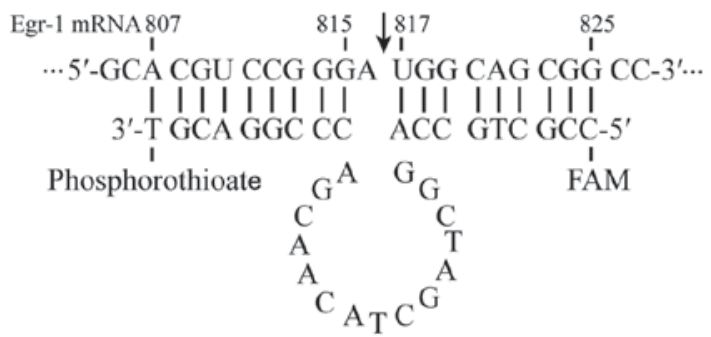

Figure 1. Construction conceptual diagram of Egr-1 DNA enzyme shear and its substrate. Egr-1, early growth response factor-1; FAM, fluorescein.

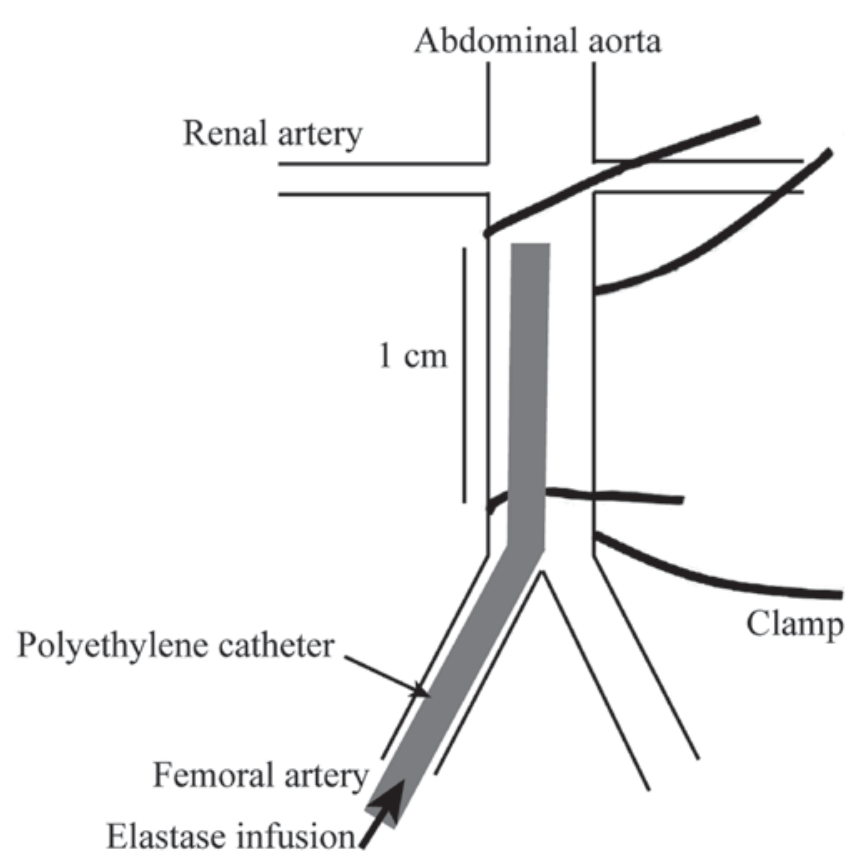

Figure 2. Schematic of the abdominal aortic aneurysm model, which used intraluminal elastase infusion.

overnight at $4^{\circ} \mathrm{C}$. Horseradish peroxidase-labeled goat antirabbit IgG (Peroxidase-Conjugated Goat anti-Rabbit IgG, cat. no. ZB-2301, 1:2,000; ZSGB-BIO) was used. Image analysis was conducted using Image lab software (Bio-Rad Laboratories, Inc., Hercules, CA, USA).

Immunohistochemistry. The specimens were fixed with $4 \%$ formalin. The slices were immersed in dewaxing xylene and ethanol. The sections were incubated in $3 \% \mathrm{H}_{2} \mathrm{O}_{2}$ for $10 \mathrm{~min}$ to inhibit endogenous peroxidase activity. These sections were blocked with $5 \%$ bovine serum albumin or milk powder. Egr-1 antibody [EGR1 (15F7) Rabbit mAb, cat. no. 4153, 1:1,000; Cell Signaling Technology, Inc.], MMP-2 (MMP-2 Polyclonal Antibody, cat. no. A6247, 1:1,000; ABclonal Technology), and MMP-9 (MMP-9 Polyclonal Antibody, cat. no. A11147, 1:1,000; ABclonal Technology) were incubated at $4^{\circ} \mathrm{C}$ overnight. After washing 3 times, biotin-labeled secondary antibody was added and incubated for $30 \mathrm{~min}$. The three membranes were washed and incubated with biotin-horseradish-peroxidase complex at room temperature for $30 \mathrm{~min}$. The films were then washed with PBS and DAB, counterstained with hematoxylin, and mounted with neutral balata. As a negative control, the primary antibody was substituted with PBS.

Statistical analysis. Data were expressed as the mean \pm standard deviation or $\chi^{2}$ test for the difference between groups. Three or more groups were compared by one-way analysis of variance and Bonferroni post hoc test for multiple comparisons. A Student's t-test was used for comparisons between 2 groups. Data were analyzed with Prism GraphPad 6 (GraphPad, Inc., La Jolla, CA, USA). P<0.05 was considered to indicate a statistically significant difference.

\section{Results}

EDRz transfection. Blood vessels were collected $24 \mathrm{~h}$ and 28 days after transfection, and transfection was confirmed by fluorescence microscopy. In the control group and jetPRIME group, no fluorescence was detected. A wide range of green fluorescence was observed in the intima and media of the blood vessels in EDRz the group. This result demonstrates that EDRz was transfected into the inner and media (Fig. 3Aa). After 28 days of transfection, green fluorescence was observed in the vessel wall, indicating that EDRz was a persistent transfection process (Fig. 3Ab).

Transmission electron microscopy. Transmission electron microscopy showed that the medial layer of the vessels was disordered and that elastic fibers were broken and arranged irregularly. The phenotype of smooth muscle cells was secretory. Moreover, smooth muscle cells were significantly reduced and irregularly arranged in the control group (Fig. 3Ba) compared to in the EDRz group (Fig. 3Bb).

EDRz inhibits the formation of AAA. AAA is a dilatation of the abdominal aorta, typically above the normal diameter of the artery by more than $50 \%$. By measuring the diameter of the abdominal aorta, all arteries reached the diagnostic criteria for aneurysms. After 28 days, elastase was perfused into the abdominal aorta in rats to determine the inhibitory effect of EDRz on AAA development by observing the aortic diameter in the EDRz group.

Aneurysm formation in the abdominal aorta at 28 days was observed in each group. Representative macroscopic images immediately before AAA induction (Normal: Fig. 4Aa) and 28 days after AAA induction in the groups are shown in Fig. 4A. The EDRz group (Fig. 4Ad) showed minimal aneurysm formation compared to the control (Fig. 4Ab) and transfection reagent group (Fig. 4Ac). The abdominal aortic diameter in the three groups gradually increased after AAA induction, with significantly smaller diameters in the EDRz group $(2.5 \pm 0.1 \mathrm{~mm})$ than in the control group $(3.5 \pm 0.1 \mathrm{~mm})$ and transfection reagent group $(3.6 \pm 0.1 \mathrm{~mm})$. No significant difference was observed between the control and transfection reagent group ( $\mathrm{P}>0.05$; Fig. $4 \mathrm{~B})$.

$H \& E$ staining. We histologically examined the thickness of the medial membrane of the artery (Fig. 5). H\&E staining showed that the number of smooth muscle cells per unit area decreased in the control group (Fig. 5A) and jetPRIME group (Fig. 5B), and the medial was obviously thinner than in the 

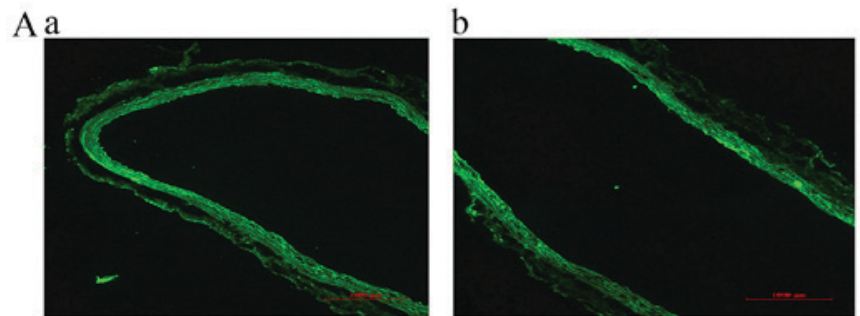

$\mathrm{B}$ a

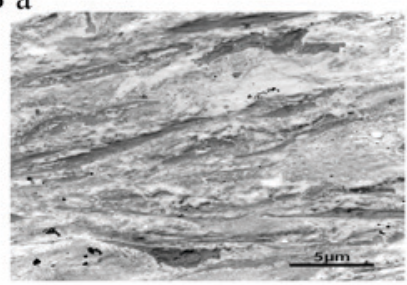

b

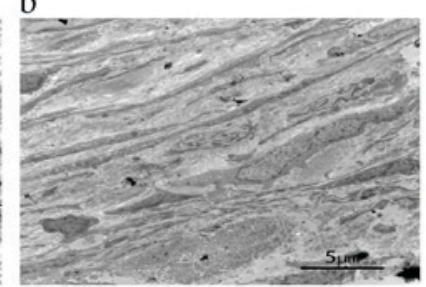

Figure 3. (Aa) Depicts a large amount of green fluorescence in the intima and media following $24 \mathrm{~h}$ of EDRz transfection, while in (Ab), green fluorescence is still evident in the arterial wall following 28 days of transfection. Scale bars $=1,000 \mu \mathrm{m}$. (B) Images representing the ultrastructure of the abdominal aorta in (Ba) control and (Bb) EDRz groups 28 days following elastase perfusion. Scale bars $=5 \mu \mathrm{m}$. EDRz, early growth response factor-1 DNA enzyme.

EDRz treatment group (Fig. 5C). The media layer thickness of the control group was slightly lower in the EDRz group (control group: $72.8 \pm 18.3 \mu \mathrm{m}$, EDRz group: $125.2 \pm 20.8 \mu \mathrm{m} ; \mathrm{P}<0.05)$. No significant difference was observed between the control and transfection reagent group (control group: $72.8 \pm 18.3 \mu \mathrm{m}$, transfection reagent group: $76.3 \pm 22.8 \mu \mathrm{m} ; \mathrm{P}>0.05$ ).

Egr-1, MMP-2, and MMP-9 mRNA levels. Compared to the control group, the EDRz group showed significantly lower mRNA expression levels of Egr-1 (control group: 1.00 \pm 0.23 , EDRz group: $0.46 \pm 0.09, \mathrm{P}<0.01$ ) and MMP-2 (control group: $1.01 \pm 0.09, \mathrm{EDRz}$ group: $0.39 \pm 0.17 ; \mathrm{P}<0.01)$ and MMP-9 (control group: $1.00 \pm 0.08$, EDRz group: 0.34 \pm 0.53 ; $\mathrm{P}<0.01)$. Compared to the jetPRIME group, the EDRz group showed significantly lower mRNA expression levels of Egr-1 (jetPRIME group: 1.09 \pm 0.24 , EDRz group: 0.46 $\pm 0.09, \mathrm{P}<0.01$ ) and MMP-2 (jetPRIME group: $1.08 \pm 0.24, \mathrm{EDRz}$ group: $0.39 \pm 0.17, \mathrm{P}<0.01$ ) and MMP-9 (jetPRIME group: $0.95 \pm 0.15$, EDRz group: $0.34 \pm 0.53, \mathrm{P}<0.01)$. The expression levels of Egr-1, MMP-2, and MMP-9 in the control and transfection reagent group did not differ significantly ( $\mathrm{P}>0.05$; Fig. 6).

Western blotting analysis. Based on the western blotting results, Egr-1 protein expression levels in the control group and jetPRIME group were significantly higher than in the EDRz group (control group: $0.72 \pm 0.10$, jetPRIME group: 0.75 \pm 0.09, EDRz group: 0.35 \pm 0.06 ) (Fig. 7A). MMP-2 protein expression levels in the control and jetPRIME group were significantly higher than in the EDRz group (control group: $1.05 \pm 0.17$, jetPRIME group: $1.00 \pm 0.13$, EDRz group: $0.63 \pm 0.10$ ) (Fig. 7B). MMP-9 protein expression levels in the control and jetPRIME group were significantly higher than in the EDRz group (control group: $1.04 \pm 0.18$, jetPRIME group: $0.93 \pm 0.08$, EDRz group: 0.53 \pm 0.11 ) (Fig. 7C). However, the expression levels of Egr-1, MMP-2, and MMP-9 in the control group and jetPRIME group did not differ significantly ( $\mathrm{P}>0.05$; Fig. 7$)$.
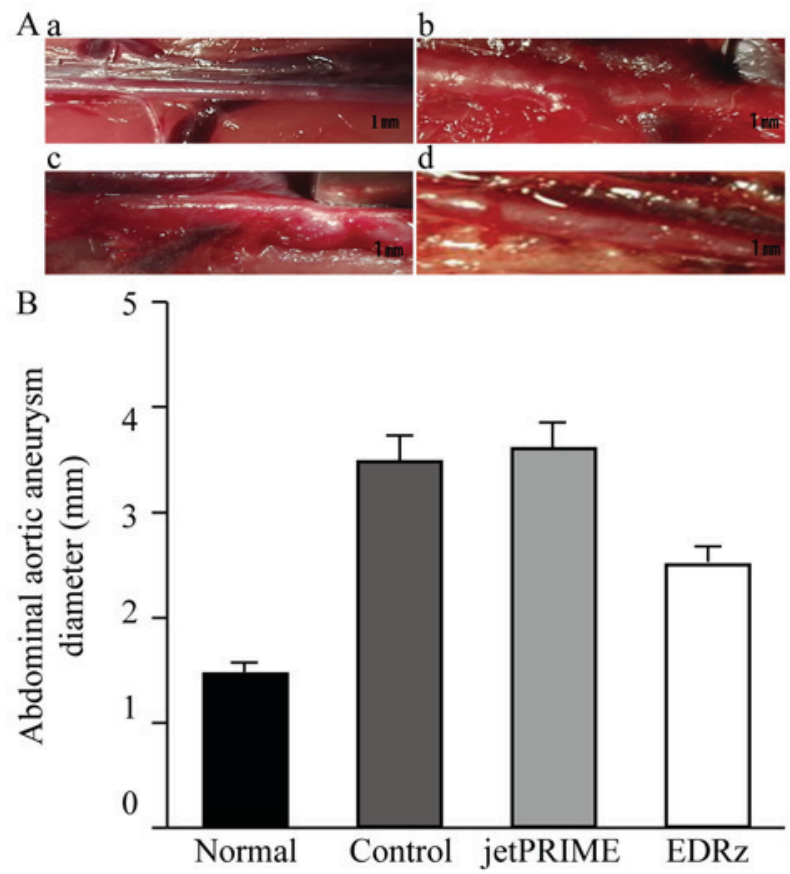

Figure 4. (A) Aneurysm formation in the abdominal aorta in 28 days is evident in each group: (Aa) normal; (Ab) control group; (Ac) jetPRIME group; and (Ad) EDRz group. Scale bars $=1 \mathrm{~mm}$. (B) Time course of the abdominal aortic diameter by ultrasound imaging. Values are presented as the mean \pm standard deviation. EDRz, early growth response factor-1 DNA enzyme.

Immunohistochemistry. Immunostaining for Egr-1 revealed a significantly higher proportion of positive area in the control group (29.3 $\pm 4.3 \%$; Fig. 8Aa) and jetPRIME group $(27.1 \pm 5.0 \%$; Fig. 8Ab) than in the EDRz group $(14.3 \pm 3.3 \%$, $\mathrm{P}<0.05$; Fig. 8Ac). MMP-2 showed a significantly higher proportion of positive area in the control group $(24.2 \pm 5.8 \%$; Fig. $8 \mathrm{Ba})$ and jetPRIME group $(24.9 \pm 6.2 \%$; Fig. $8 \mathrm{Bb})$ than in the EDRz group $(11.3 \pm 2.6 \%$; $\mathrm{P}<0.05$; Fig. 8Bc). MMP-9 showed a significantly higher proportion of positive area in the control group (20.8 $\pm 3.3 \%$; Fig. $8 \mathrm{Ca})$ and jetPRIME group $(21.1 \pm 5.3 \%$; Fig. $8 \mathrm{Cb})$ than in the EDRz group $(14.1 \pm 6.3 \%$, $\mathrm{P}<0.05$; Fig. 8Cc). However, the expression levels of Egr-1, MMP-2, and MMP-9 in the control and jetPRIME group did not differ significantly ( $\mathrm{P}>0.05$; Fig. 8).

\section{Discussion}

Egr-1, a zinc finger early transcription factor, is a member of the early gene family. In the study of AAA, Egr-1 has attracted much attention. Egr-1 is poorly expressed in the normal artery wall. However, it is induced by acute mechanical injury, other vascular stresses, growth factors, and proinflammatory cytokines $(12,13)$. Egr-1 also regulates the expression of genes related to the development of vascular diseases (14). Bioinformatics analysis showed that Egr-1 is a key transcription factor in the formation and pathogenesis of AAA (15). Egr-1 was found to be involved in thrombus formation and the inflammatory pathogenesis of AAA. The role of Egr-1 as a mechanical stress-response gene that causes aneurysm formation was also demonstrated (11). Thus, Egr-1 is being considered as a new target for AAA prevention and control. 
A

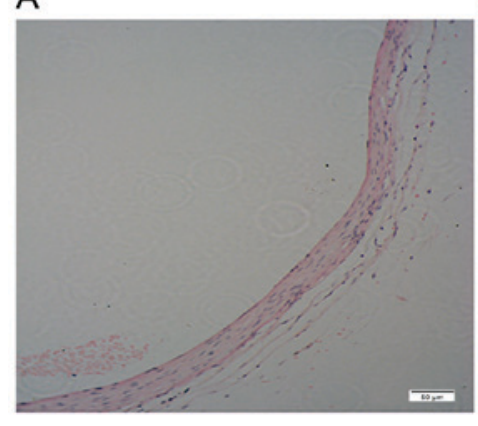

B

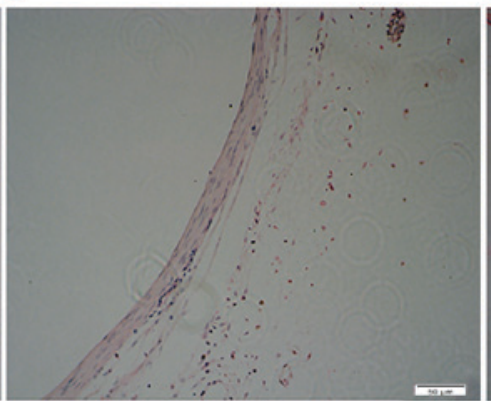

C

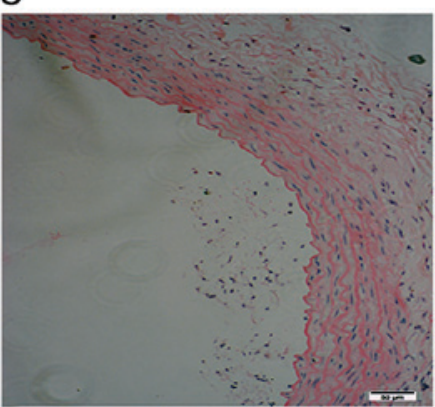

Figure 5. Hematoxylin and eosin staining revealed the morphological alterations in abdominal aortic aneurysm. (A) Control group, (B) jetPRIME group and (C) early growth response factor-1 DNA enzyme group. Scale bars=50 $\mu \mathrm{m}$.
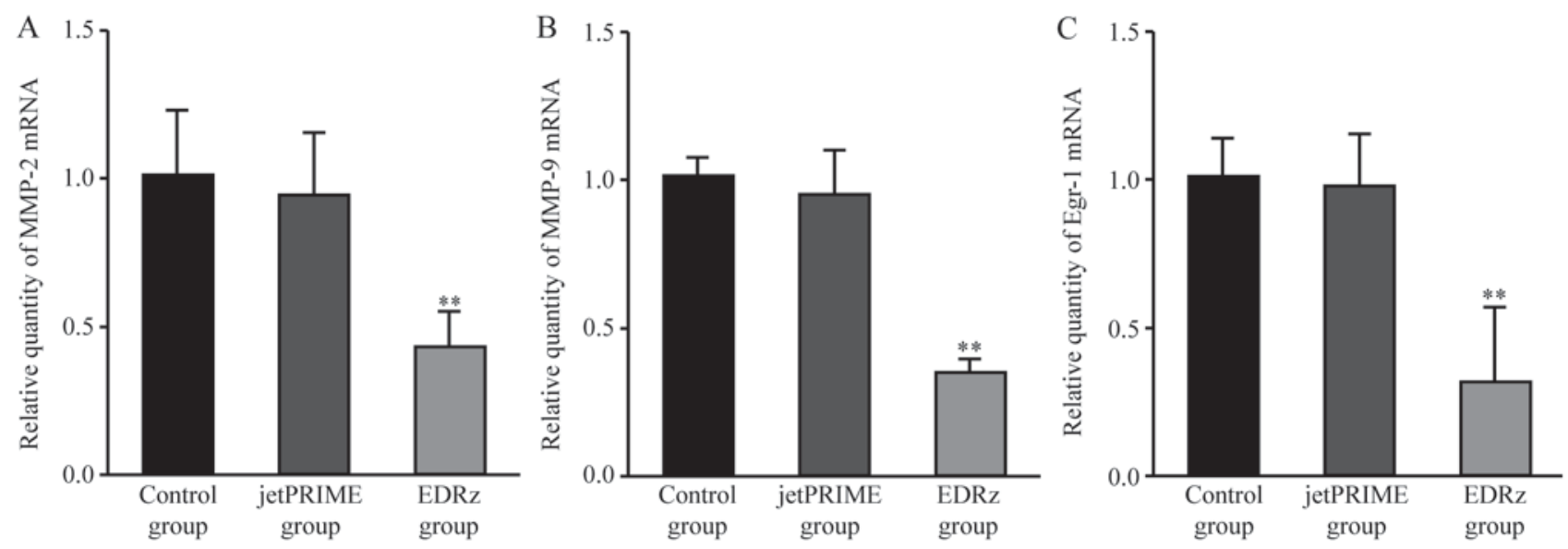

Figure 6. Expression of mRNA was detected by reverse transcription-quantitative polymerase chain reaction. Graphs representing the levels of (A) MMP-2 mRNA expression, (B) MMP-9 mRNA expression and (C) Egr-1 mRNA expression. ${ }^{* *} \mathrm{P}<0.01$ vs. control. MMP, matrix metalloproteinase; Egr-1, early growth response factor-1.
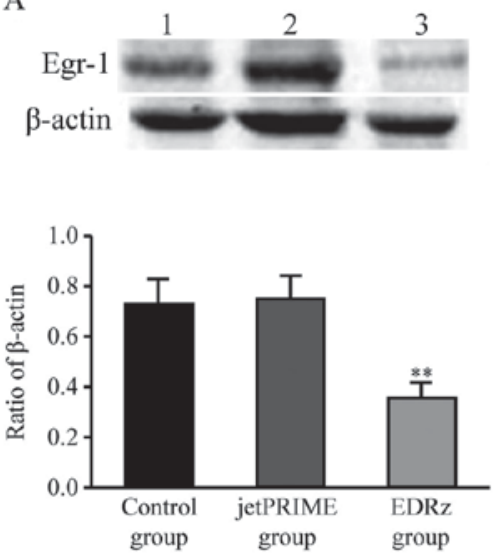

B
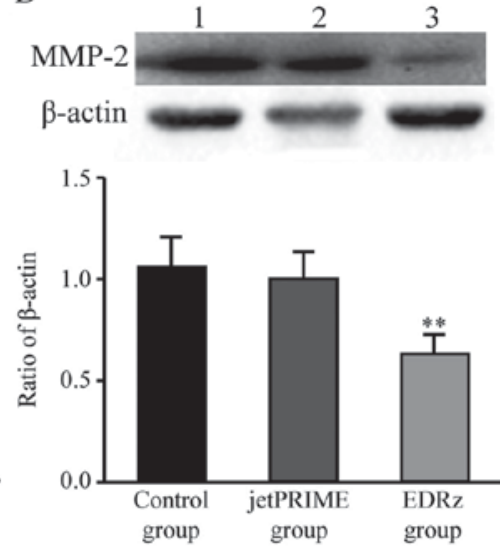

C
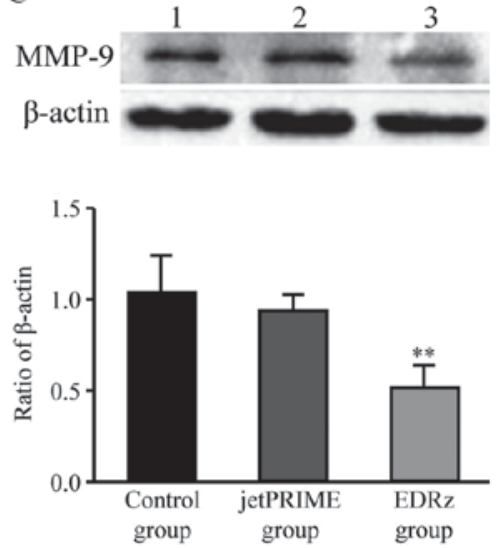

Figure 7. Western blotting results of Egr-1, MMP-2 and MMP-9 protein expressions. Results are presented as the mean \pm standard deviation. (A) Egr-1, (B) MMP-2 and (C) MMP-9 protein expression. ${ }^{* *} \mathrm{P}<0.01$ vs. control group. Lane 1, Control group; lane 2, jetPRIME group; lane 3, EDRz group. MMP, matrix metalloproteinase; Egr-1, early growth response factor-1; EDRz, early growth response factor-1 DNA enzyme.

DNA enzymes (deoxyribozyme, DRz) are DNA molecules with catalytic capabilities that cleave specific RNA strands. The '10-23' DNA enzyme is the 23rd clone of the 10th cycle of in vitro selection. Therefore, the enzyme activity center is a '10-23 motif' (16,17). AUG (816-818 sequence) is a selected target of Egr-1 mRNA. A phosphorothioate modification was made in the $3^{\prime}$ end to resist nuclease degradation, and the $5^{\prime}$ end was labeled with carboxy fluorescein for detection purposes. Base 816 (A) of the Egr-1 mRNA did not base pair with EDRz. However, the remaining EDRz sites base paired with Egr-1 mRNA, followed by conformational changes. Moreover, nucleophilic attack occurred on the adjacent phosphate. The 
A a

$\mathrm{b}$ c
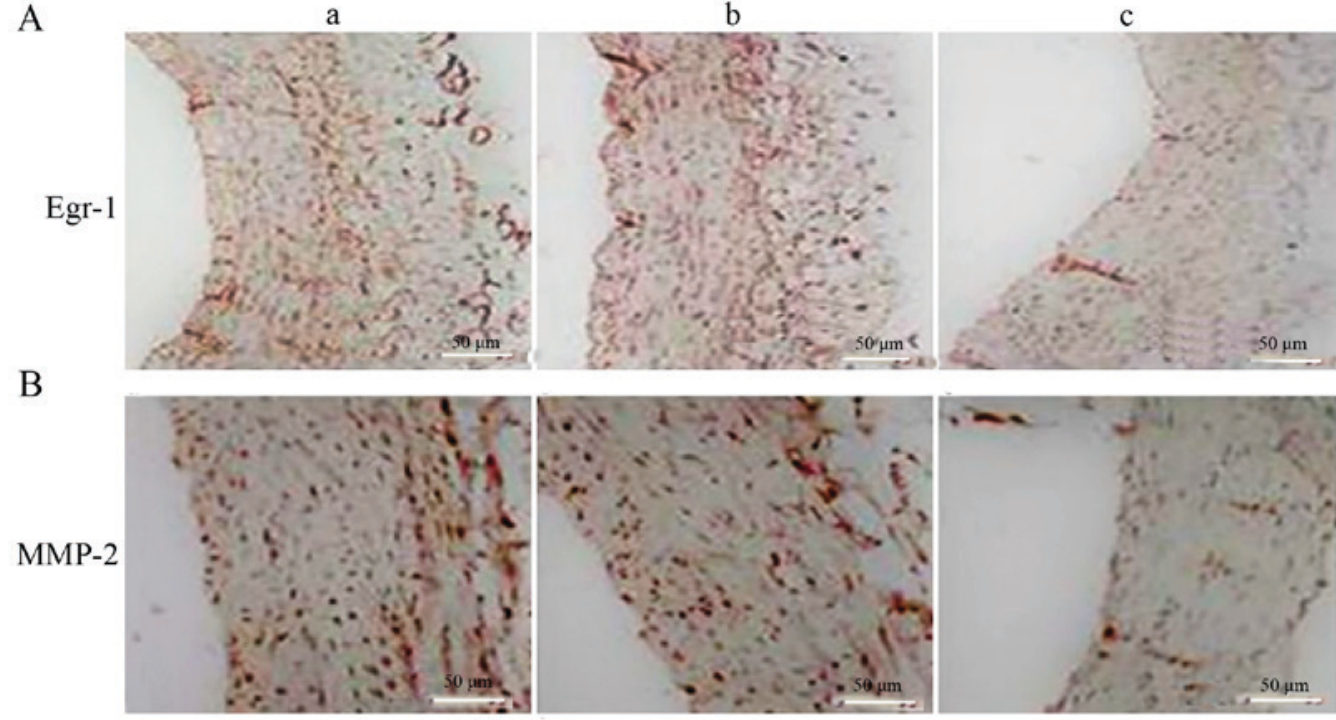

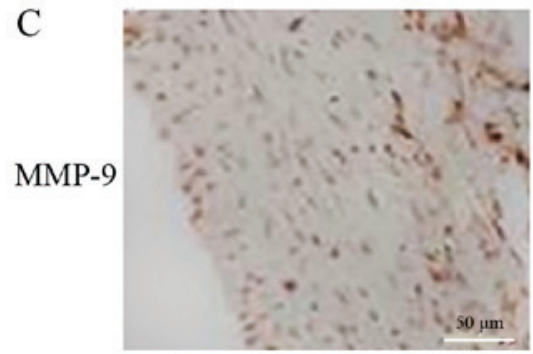

Control group

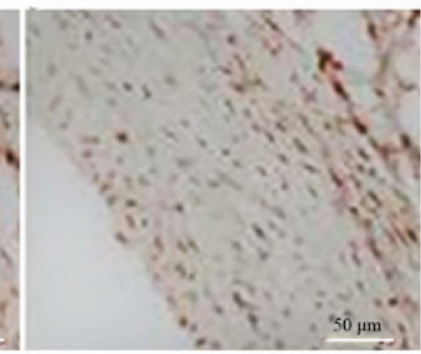

jetPRIME group

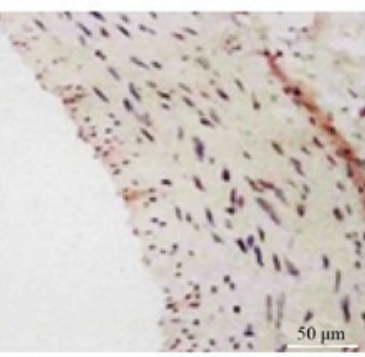

$\mathrm{EDRz}$ group

Figure 8. Immunohistochemistry results of Egr-1, MMP-2 and MMP-9 protein expressions. (A) Egr-1, (B) MMP-2 and (C) MMP-9 expression. Image (a) Control group; image (b), jetPRIME group; and image (c), EDRz group. Yellow staining was positive expression and the fluorescence gray value per unit area was measured (scale bars, $50 \mu \mathrm{m}$ ). MMP, matrix metalloproteinase; Egr-1, early growth response factor-1; EDRz, early growth response factor-1 DNA enzyme.

Egr-1 mRNA molecular structure was dissociated by two transesterification reactions. Our previous study demonstrated the specificity and efficacy of synthetic EDRz for inhibiting Egr-1 $(7,18,19)$. However, whether the development of AAA can be suppressed by inhibiting Egr-1 expression remains unknown.

Synthetic DNA enzymes that specifically degrade Egr-1 mRNA prevent the induction of Egr-1 protein expression in human and rat aortic vascular smooth muscle cells (20-22). In previous studies, Egr-1 DNA enzyme reduced the mRNA and protein expression of Egr-1 in aortic smooth muscle cells and inhibited cell proliferation and migration (23). This property has been successfully applied to suppress gene expression. In our study, green fluorescence was observed in the vessel wall after $24 \mathrm{~h}$ and 28 days, indicating that EDRz was a persistent transfection process and that expression of Egr-1 was continuously inhibited. EDRz can effectively inhibit the formation and development of AAA. AAA is a dilatation of the abdominal aorta, typically above the normal diameter of the artery by more than 50\%. By measuring the diameter of the abdominal aorta, all arteries reached the diagnostic criteria for aneurysms. The EDRz group showed minimal aneurysm formation compared to the control and transfection reagent group. These results suggest that EDRz inhibits AAA by inhibiting Egr-1 expression.

Elastase perfusion degrades the elastic fiber in the media and forms elastic fragments. In our study, H\&E staining and transmission electron microscopy showed that the medial layer of the vessels was disordered and that elastic fibers were broken and arranged irregularly in the control group and jetPRIME group. In the control group, the media was significantly thinner than in the EDRz group.

Moreover, the number of smooth muscle cells was significantly reduced and these cells were irregularly arranged in the control group compared to in the EDRz group. This causes a local inflammatory reaction and produces proinflammatory factors, MMPs, endogenous and elastic proteases, among others. These processes promote elastase and collagen fiber degradation. Arterial wall inflammation can cause vascular smooth muscle cell apoptosis, MMP expression, and inflammation. Therefore, MMPs promote the degradation of the extracellular matrix, and inflammation participates in the occurrence and development of AAA $(24,25)$. Moreover, the suppression of MMP expression (26) can effectively inhibit AAA. Destruction of the elastic plate in blood vessels also contributes to cell proliferation, migration, and relocalization in the extracellular matrix, which promotes arterial expansion and causes matrix shrinkage and aneurysm formation and rupture. MMPs are proteases that can promote extracellular matrix degradation and an inflammatory response, both of which contribute to aneurysm development. Particularly, MMP-2 and MMP-9 can significantly degrade elastin and participate in inflammatory responses (27). 
Egr-1, MMP-2, and MMP-9 levels were evaluated to determine the effectiveness of EDRz in inhibiting the AAA response. In the present study, the mRNA and protein expression levels of Egr-1, MMP-2, and MMP-9 were determined along with the inhibition of AAA genesis in rats transfected with EDRz. The results indicate that EDRz inhibits AAA development and reduces MMP-9 and MMP-2 expression.

Previous studies showed that Egr-1 promotes the expression of MMP-9 and MMP-2 and the migration and metastasis of inflammatory and cancer cells. Moreover, this phenomenon causes the formation and rupture of aneurysms (28). Shin et al (29) found that MMP2 and MMP-9 promote the degradation of elasticity and extracellular matrix and inflammation. MMP-9 transcription caused by tumor necrosis factor is closely related to Egr-1. MMP-9 expression activity is enhanced by Egr-1 binding to its promoter. Harja et al (30) observed that the MMP-2 expression level in apo $\mathrm{E}^{-/-}$knockout mice was higher than that in C56BL6 mice with the same genetic background. Although it remains unclear whether Egr-1 can bind to MMP-2, a previous study demonstrated that stimulation of MMP-2 is related to the expression of MT1-MMP. Egr-1 can be combined with the MT1-MMP binding site (31). Sho et al (32) reported that mechanical and shear stresses can induce Egr-1 expression, whereas the transcription of MT1-MMP regulated by Egr-1 can activate MMP-2. MT1-MMP is found in endothelial and smooth muscle cells, which may be involved in long-term maintenance of MMP-2 activation and further contribute to dilatation of the aorta. Therefore, EDRz may inhibit the development of AAA by inhibiting MMP-2 and MMP-9 expression. However, the specific mechanism of this inhibitory effect requires further investigation.

This study has some limitations. The rat model of elastase-induced AAA used in our study is pathologically different from human AAAs. Human AAAs often exhibit atherosclerosis and intramural thrombosis such as in diabetic or hyperlipidemia rats. Whether the dose of EDRz used in this study was appropriate is unclear. We selected the EDRz dose based on our previous report, which showed that EDRz prevents stenosis and occlusion of autogenous vein graft in vivo. How to apply this information in humans also remains unclear. Whether EDRz can delay or treat AAA progression when administrated after the onset of AAA is unknown, which will be the focus of our future studies.

In conclusion, we found that Egr-1 plays an important role in AAA formation. EDRz inhibits the mRNA and protein expression of Egr-1 and regulates the expression of MMP-2 and MMP-9. Thus, the development of AAA in rats was inhibited. EDRz may be useful as a new drug for preventing or treating AAA.

\section{Acknowledgements}

Not applicable.

\section{Funding}

The present study was supported by the Nature Science Foundation of China (grant nos. 30801123 and 81070254) and the Reserve Talents of Universities Overseas Research Program of Heilongjiang and Project Sponsored by the Scientific Research Foundation for the Returned Overseas Chinese Scholars, State Education Ministry [grant no. (2014) 1685].

\section{Availability of data and materials}

All data generated or analyzed during this study are included in the published article.

\section{Authors' contribution}

SW and HD contributed equally to this paper. SW, HD, CL, $\mathrm{XH}$ and $\mathrm{YF}$ were involved in the study conception and design. SW, HD, GX and CO were involved in analysis and interpretation of data. SW, HD, GX and LC collected data. SW, HD, $\mathrm{CL}, \mathrm{GX}, \mathrm{XH}, \mathrm{CO}$ and LC wrote the article. CL, XH, CO and YF performed critical revision of the article. SW, HD, CL, GX, XH and CO approved the final article. SW, HD, GX, LC and YF performed statistical analysis. CL and XH obtained funding. CL had overall responsibility.

\section{Ethics approval and consent to participate}

The animal use protocol was been reviewed and approved by the Institutional Animal Care and Use Committee (IACUC) of Jiamusi University (Heilongjiang, China).

\section{Consent for publication}

Not applicable.

\section{Competing interests}

The authors declare there they have no competing interest.

\section{References}

1. Grøndal N, Søgaard R and Lindholt JS: Baseline prevalence of abdominal aortic aneurysm, peripheral arterial disease and hypertension in men aged 65-74 years from a population screening study (VIVA trial). Br J Surg 102: 902-906, 2015.

2. Benson RA, Poole R, Murray S, Moxey P and Loftus IM: Screening results from a large United Kingdom abdominal aortic aneurysm screening center in the context of optimizing United Kingdom national abdominal aortic aneurysm screening programme protocols. J Vasc Surg 63: 301-304, 2016.

3. Shin S, Cho YP, Jun H, Park H, Hong HN and Kwon TW: Transglutaminase type 2 in human abdominal aortic aneurysm is a potential factor in the stabilization of extracellular matrix. J Vasc Surg 57: 1362-1370, 2013.

4. Tang L, Cong Z, Hao S, Li P, Huang H, Shen Y, Li K and Jing H: Protective effect of melatonin on the development of abdominal aortic aneurysm in a rat model. J Surg Res 209: 266-278.e1, 2017.

5. Pagel JI and Deindl E: Early growth response 1-a transcription factor in the crossfire of signal transduction cascades. Indian $\mathbf{J}$ Biochem Biophys 48: 226-235, 2011.

6. Wang NP, Pang XF, Zhang LH, Tootle S, Harmouche S and Zhao ZQ: Attenuation of inflammatory response and reduction in infarct size by postconditioning are associated with downregulation of early growth response 1 during reperfusion in rat heart. Shock 41: 346-354, 2014.

7. Liu C, Zhang X, Wang S, Cheng M, Liu C, Wang S, Hu X and Zhang Q: Transfected early growth response gene-1 DNA enzyme prevents stenosis and occlusion of autogenous vein graft in vivo. Biomed Res Int 2013: 310406, 2013. 
8. Ha YM, Lee DH, Kim M and Kang YJ: High glucose induces connective tissue growth factor expression and extracellular matrix accumulation in rat aorta vascular smooth muscle cells via extracellular signal-regulated kinase 1/2. Korean J Physiol Pharmacol 17: 307-314, 2013

9. Charolidi N, Pirianov G, Torsney E, Pearce S, Laing K, Nohturfft A and Cockerill GW: Pioglitazone identifies a new target for aneurysm treatment: Role of Egrl in an experimental murine model of aortic aneurysm. J Vasc Res 52: 81-93, 2015.

10. Shin IS, Kim JM, Kim KL, Jang SY, Jeon ES, Choi SH, Kim DK, Suh W and Kim YW: Early growth response factor-1 is associated with intraluminal thrombus formation in human abdominal aortic aneurysm. J Am Coll Cardiol 53: 792-799, 2009.

11. Yamashiro Y, Papke CL, Kim J, Ringuette LJ, Zhang QJ, Liu ZP, Mirzaei H, Wagenseil JE, Davis EC and Yanagisawa H: Abnormal mechanosensing and cofilin activation promote the progression of ascending aortic aneurysms in mice. Sci Signal 8: ra105, 2015.

12. Kong L, Shen X, Lin L, Leitges M, Rosario R, Zou YS and Yan SF: PKC $\beta$ promotes vascular inflammation and acceleration of atherosclerosis in diabetic ApoE null mice. Arterioscler Thromb Vasc Biol 33: 1779-1787, 2013.

13. Morawietz H, Ma YH, Vives F, Wilson E, Sukhatme VP, Holtz J and Ives HE: Rapid induction and translocation of Egr-1 in response to mechanical strain in vascular smooth muscle cells. Circ Res 84: 678-687, 1999.

14. Fu M, Zhu X, Zhang J, Liang J, Lin Y, Zhao L, Ehrengruber MU and Chen YE: Egr-1 target genes in human endothelial cells identified by microarray analysis. Gene 315: 33-41, 2003.

15. Yuan K, Liang W and Zhang J: A comprehensive analysis of differentially expressed genes and pathways in abdominal aortic aneurysm. Mol Med Rep 12: 2707-2714, 2015.

16. Li J, Wang N, Luo Q and Wan L: The 10-23 DNA enzyme generated by a novel expression vector mediate inhibition of taco expression in macrophage. Oligonucleotides 20: 61-68, 2010.

17. Lam CH and Perrin DM: Introduction of guanidinium-modified deoxyuridine into the substrate binding regions of DNAzyme 10-23 to enhance target affinity: Implications for DNAzyme design. Bioorg Med Chem Lett 20: 5119-5122, 2010.

18. Pagel JI, Ziegelhoeffer T, Heil M, Fischer S, Fernández B, Schaper W, Preissner KT and Deindl E: Role of early growth response 1 in arteriogenesis: impact on vascular cell proliferation and leukocyte recruitment in vivo. Thromb Haemost 107: $562-574,2012$

19. Dickinson MG, Kowalski PS, Bartelds B, Borgdorff MA, van der Feen D, Sietsma H, Molema G, Kamps JA and Berger RM: A critical role for Egr-1 during vascular remodelling in pulmonary arterial hypertension. Cardiovasc Res 103: 573-584, 2014.

20. Huang M, Satchell L, Duhadaway JB, Prendergast GC and Laury-Kleintop LD: RhoB links PDGF signaling to cell migration by coordinating activation and localization of $\mathrm{Cdc} 42$ and Rac. J Cell Biochem 112: 1572-1584, 2011.

21. Uchida K, Sasahara M, Morigami N, Hazama F and Kinoshita M: Expression of platelet-derived growth factor B-chain in neointimal smooth muscle cells of balloon injured rabbit femoral arteries. Atherosclerosis 124: 9-23, 1996.
22. Iyoda T, Zhang F, Sun L, Hao F, Schmitz-Peiffer C, Xu X and Cui MZ: Lysophosphatidic acid induces early growth response-1 (Egr-1) protein expression via protein kinase C $\delta$-regulated extracellular signal-regulated kinase (ERK) and c-Jun N-terminal kinase (JNK) activation in vascular smooth muscle cells. J Biol Chem 287: 22635-22642, 2012.

23. Wu Y, Han W and Liu GN: A DNA enzyme targeting Egr-1 inhibits rat vascular smooth muscle cell proliferation by down-regulation of cyclin D1 and TGF-beta1. Braz J Med Bio Res 43: 17-24, 2010.

24. Longo GM, Xiong W, Greiner TC, Zhao Y, Fiotti N and Baxter BT: Matrix metalloproteinases 2 and 9 work in concert to produce aortic aneurysms. J Clin Invest 110: 625-632, 2002

25. Yan YF, Pei JF, Zhang Y, Zhang R, Wang F, Gao P, Zhang ZQ, Wang TT, She ZG, Chen HZ and Liu DP: The paraoxonase gene cluster protects against abdominal aortic aneurysm formation. Arterioscler Thromb Vasc Biol 37: 291-300, 2017.

26. Chabasse C, Siefert SA, Chaudry M, Hoofnagle MH, Lal BK and Sarkar R: Recanalization and flow regulate venous thrombus resolution and matrix metalloproteinase expression in vivo. J Vasc Surg Venous Lymphat Disord 3: 64-74, 2015.

27. Tanaka A, Hasegawa T, Chen Z, Okita Y and Okada K: A novel rat model of abdominal aortic aneurysm using a combination of intraluminal elastase infusion and extraluminal calcium chloride exposure. J Vasc Surg 50: 1423-1432, 2009.

28. Pezet M, Jacob MP, Escoubet B, Gheduzzi D, Tillet E, Perret P, Huber P, Quaglino D, Vranckx R, Li DY, et al: Elastin haploinsufficiency induces alternative aging processes in the aorta. Rejuvenation Res 11: 97-112, 2008.

29. Shin SY, Kim JH, Baker A, Lim Y and Lee YH: Transcription factor Egr-1 is essential for maximal matrix metalloproteinase-9 transcription by tumor necrosis factor alpha. Mol Cancer Res 8: 507-519, 2010.

30. Harja E, Chang JS, Lu Y, Leitges M, Zou YS, Schmidt AM and Yan SF: Mice deficient in PKCbeta and apolipoprotein E display decreased atherosclerosis. FASEB J 23: 1081-1091, 2009.

31. Haas TL, Stitelman D, Davis SJ, Apte SS and Madri JA: Egr-1 mediates extracellular matrix-driven transcription of membrane type 1 matrix metalloproteinase in endothelium. J Biol Chem 274: 22679-22685, 1999.

32. Sho E, Sho M, Singh TM, Nanjo H, Komatsu M, Xu C, Masuda $\mathrm{H}$ and Zarins CK: Arterial enlargement in response to high flow requires early expression of matrix metalloproteinases to degrade extracellular matrix. Exp Mol Pathol 73: 142-153, 2002.

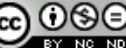

This work is licensed under a Creative Commons Attribution-NonCommercial-NoDerivatives 4.0 International (CC BY-NC-ND 4.0) License. 